Article

\title{
Nonclassical Symmetries of a Nonlinear Diffusion-Convection/Wave Equation and Equivalents Systems
}

\author{
Daniel J. Arrigo, Brandon P. Ashley, Seth J. Bloomberg and Thomas W. Deatherage
}

Department of Mathematics, University of Central Arkansas, Conway, AR 72035, USA;

brandonpashley@live.com (B.A.); sbloomberg1@cub.uca.edu (S.B.); tdeatherage1@cub.uca.edu (T.D.)

* Correspondence: darrigo@uca.edu; Tel.: +501-450-5668

Academic Editor: Roman M. Cherniha

Received: 07 August 2016; Accepted: 22 November 2016; Published: 26 November 2016

\begin{abstract}
It is generally known that classical point and potential Lie symmetries of differential equations (the latter calculated as point symmetries of an equivalent system) can be different. We question whether this is true when the symmetries are extended to nonclassical symmetries. In this paper, we consider two classes of nonlinear partial differential equations; the first one is a diffusion-convection equation, the second one a wave, where we will show that the majority of the nonclassical point symmetries are included in the nonclassical potential symmetries. We highlight a special case were the opposite is true.
\end{abstract}

Keywords: nonclassical symmetry; nonclassical potential symmetry; diffusion equation; wave equation

\section{Introduction}

Symmetry analysis plays a fundamental role in the construction of exact solutions to nonlinear partial differential equations. Based on the original work of Lie [1] on continuous groups, symmetry analysis provides a unified explanation for the seemingly diverse and ad hoc integration methods used to solve ordinary differential equations. At the present time, there is extensive literature on the subject, and we refer the reader to the books by Arrigo [2], Bluman and Kumei [3], and Olver [4].

A particular class of equation that has benefited from this type of analysis is the nonlinear diffusion equation

$$
u_{t}=\left(K(u) u_{x}\right)_{x}
$$

From a symmetry point of view, this equation was first considered by Ovsjannikov [5] (see also [3] and [6]), where it was found that (1) admits nontrivial symmetries for a variety of different diffusivities. In particular, power law diffusion, where

$$
u_{t}=\left(u^{m} u_{x}\right)_{x}
$$

admits the symmetry generator

$$
\Gamma=T \frac{\partial}{\partial t}+X \frac{\partial}{\partial x}+U \frac{\partial}{\partial u}
$$

where $T, X$, and $U$ are

$$
\begin{aligned}
T & =c_{1}+c_{2} t \\
X & =c_{3}+c_{4} x \\
U & =\frac{1}{m}\left(2 c_{4}-c_{2}\right) u
\end{aligned}
$$


(where $c_{i}$ are arbitrary constants) for general powers $m(m \neq 0)$, and in the special case $m=-4 / 3$, where (2) admits an additional symmetry with generator

$$
\Gamma=x^{2} \frac{\partial}{\partial x}-3 x u \frac{\partial}{\partial u}
$$

In 1988, Bluman, Reid, and Kumei [7] considered the equivalent system

$$
v_{t}=K(u) u_{x}, \quad v_{x}=u
$$

and found that this system possesses a rather rich symmetry structure and identified new forms of $K(u)$ that admitted new nontrivial symmetries. Of particular interest are again power law diffusivities $K(u)=u^{m}$, where (5) admits the symmetry generator

$$
\Gamma=T \frac{\partial}{\partial t}+X \frac{\partial}{\partial x}+U \frac{\partial}{\partial u}+V \frac{\partial}{\partial v}
$$

where $T, X, U$, and $V$ are given by, in the case of $m \neq-2$,

$$
\begin{aligned}
T & =c_{1}+c_{2} t \\
X & =c_{3}+\frac{c_{2}+m c_{4}}{m+2} x \\
U & =\frac{2 c_{4}-c_{2}}{m+2} u \\
V & =c_{5}+c_{4} v
\end{aligned}
$$

and in the case of $m=-2$,

$$
\begin{aligned}
T & =c_{1}+2 c_{2} t+4 c_{3} t^{2} \\
X & =\left(c_{6}-2 c_{3} t-c_{5} v-c_{3} v^{2}\right) x+F(t, v) \\
U & =\left(c_{2}-c_{6}+6 c_{3} t+c_{5} v+c_{3} v^{2}\right) u+\left(c_{5} x+2 c_{3} x v-F_{v}\right) u^{2} \\
V & =c_{4}+2 c_{5} t+\left(c_{2}+4 c_{3} t\right) v
\end{aligned}
$$

where $F$ satisfies $F_{t}=F_{v v}$. Clearly, the powers $m=-4 / 3$ and $m=-2$ show themselves as special, and-as this example demonstrates-the symmetries of equations and equivalent systems can be different. A natural question to ask is whether this holds true for nonclassical symmetries; that the nonclassical symmetries of a particular equation and a equivalent system (nonclassical potential symmetries) are different.

The nonclassical method, first introduced by Bluman and Cole [8] (see, for example, [2] or [3]), seeks invariance of a given partial differential equation (PDE) augmented with the invariant surface condition. As the determining equations for these nonclassical symmetries are nonlinear, there seemed to be little hope for this new method; however, with the development of computer algebra systems, the nineties saw a huge explosion of interest as several authors took interest in the nonclassical method and continues today to be an active area of interest (e.g., [9-23] and references within).

Of particular interest here is the paper by Bluman and Yan [24]. They consider two algorithms that extend the nonclassical method to potential systems and potential equations. They consider the nonlinear diffusion Equation (1), an equivalent potential system (Algorithm 1)

$$
v_{x}=u, \quad v_{t}=K(u) u_{x}
$$

and potential equation (Algorithm 2)

$$
v_{t}=K\left(v_{x}\right) v_{x x}
$$


In the case where $K(u)=\frac{1}{u^{2}+u}$, they were able to show that (10) admits nonclassical symmetries that the original Equation (1) does not. So, there is some evidence that the nonclassical symmetries of a $\mathrm{PDE}$ and a potential equation/equivalent system can be different (see also [25] and references within). Although we will not address this question in general here, we will use Algorithm 1 to consider a large class of nonlinear diffusion-convection and wave equations to show that-in the majority of cases-the nonclassical potential system symmetries contain the nonclassical symmetries of the original equation. We also highlight a special case where the opposite is true.

\section{Nonclassical Symmetries}

In this section, we consider the nonclassical symmetries of the following nonlinear partial differential equations

$$
\begin{aligned}
\text { (i) } u_{t} & =\left(F(u) u_{x}+G(u)\right)_{x} \\
\text { (ii) } u_{t t} & =\left(F(u) u_{x}+G(u)\right)_{x}
\end{aligned}
$$

These equations are of considerable interest because of their applications. For example, (11a), sometimes known as Richard's equation, has been used to model the one-dimensional, nonhysteretic infiltration in uniform nonswelling soil (Broadbridge and White [26]) and to model two phase filtration under gravity (Rogers, Stallybrass, and Clement [27]). Furthermore, (11b)—sometimes known as the nonlinear telegraph equation-has been used to model the telegraphy of a two-conductor transmission line (Katayev [28]) and the motion of a hyperelastic homogeneous rod whose cross-sectional area varies exponentially along the rod (Jeffery [29]).

In what follows, we omit the cases where (11) are linear or linearizable via a point transformation, as it is known that all solutions of linear PDEs can be obtained via classical Lie symmetries [30]. Each equation will be considered separately.

\subsection{Nonlinear Diffusion-Convection Equation}

We first consider the nonclassical symmetries of (11a). These are calculated by appending to (11a) the invariant surface condition

$$
T u_{t}+X u_{x}=U
$$

As usual, if $T \neq 0$, we set $T=1$ in (12) without loss of generality. This gives rise to the following determining equations for the infinitesimals $X(t, x, u)$ and $U(t, x, u)$ :

$$
\begin{aligned}
F X_{u u}-F_{u} X_{u} & =0 \\
F^{2} U_{u u}-2 F^{2} X_{x u}+F F_{u} U_{u}+\left(2 F G_{u}+2 F X\right) X_{u}+U F F_{u u}-U F_{u}^{2} & =0 \\
F U_{t}-F^{2} U_{x x}-F G_{u} U_{x}+2 F U X_{x}-U^{2} F_{u} & =0 \\
2 F^{2} U_{x u}+F X_{t}-F^{2} X_{x x}+2 F F_{u} U_{x}-2 F U X_{u}+F\left(2 X+G_{u}\right) X_{x} & \\
+\left(F G_{u u}-F_{u} G_{u}-X F_{u}\right) U & =0
\end{aligned}
$$

A variation of these determining equations are given in Cherniha and Serov [31], and in the case of $G=0$, appear in Arrigo and Hill [32]. To calculate the nonclassical potential symmetries, we calculate the nonclassical symmetries for the associated system

$$
v_{t}=F(u) u_{x}+G(u), \quad v_{x}=u
$$

augmented with the two associated invariant surface conditions

$$
T u_{t}+X u_{x}=U, \quad T v_{t}+X v_{x}=V
$$


again noting that we will set $T=1$, as we are assuming that $T \neq 0$. Our approach to obtaining the determining equations is through compatibility. Several authors have shown that this is equivalent to the nonclassical method (see [33-36]). Solving (14) and (15) for the first order derivatives $u_{t}, u_{x}, v_{t}$, and $v_{x}$ gives

$$
u_{t}=\frac{X G+U F-X V+u X^{2}}{F}, \quad u_{x}=\frac{V-u X-G}{F}, \quad v_{t}=V-u X, \quad v_{x}=u
$$

Requiring compatibility by eliminating partial derivatives by cross-differentiation gives

$$
\begin{gathered}
F V_{x}+(V-u X-G) V_{u}+u F V_{v}-u F X_{x}+u(u X+G-V) X_{u}-u^{2} F X_{v}-F U=0 \\
F V_{t}+F X V_{x}+F U V_{u}+F V V_{v}+\left(F X_{x}-2(G+u X) X_{u}-F U_{u}-U F_{u}\right) V \\
+X_{u} V^{2}-F^{2} U_{x}+F(u X+G) U_{u}-u F^{2} U_{v}-u F X_{t}-F(2 u X+G) X_{x} \\
+\left((u X+G)^{2}-u F U\right) X_{u}-u F(u X+G) X_{v}+(u X+G) U F_{u}-F U G_{u}-F X U=0
\end{gathered}
$$

In the case of $G=0$, these determining equations are equivalent to those that appear in Bluman and Shtelen [37]. It is interesting to note that at first appearance, (17) seems to be underdetermined-two equations for the three unknowns $X, U$ and $V$. However, if we let $V=F W+u X+G$, where $W=W(t, x, u, v)$, then (16) becomes

$$
u_{t}=U-X W, \quad u_{x}=W, \quad v_{t}=F W+G, \quad v_{x}=u
$$

and compatibility of (18) again, by cross-differentiation gives rise to the determining equations

$$
\begin{array}{r}
W_{t}+X W_{x}+U W_{u}+(F W+G+u X) W_{v}+X_{u} W^{2}+\left(X_{x}+u X_{v}-U_{u}\right) W-U_{x}-u U_{v}=0 \\
F W_{x}+W(F W+G)_{u}+u F W_{v}+X W-U=0
\end{array}
$$

To show that the nonclassical symmetries of (11a) are included in the nonclassical symmetries of (14) is to show that $V$ exists satisfying (17) if $X$ and $U$ satisfy (13). As we have defined $V$ in terms of $W$, it suffices to have $X, U$, and $W$ functions of $t, x$, and $u$ only. Doing so and requiring that (19) be compatible via cross-differentiation gives rise to

$$
\begin{array}{r}
F\left(F X_{u u}-F_{u} X_{u}\right) W^{3} \\
-\left(F^{2} U_{u u}-2 F^{2} X_{x u}+\right. \\
\left.F F_{u} U_{u}+\left(2 F G_{u}+2 F X\right) X_{u}+U F F_{u u}-U F_{u}^{2}\right) W^{2} \\
-\left(2 F^{2} U_{x u}+F X_{t}-F^{2} X_{x x}+2 F F_{u} U_{x}-2 F U X_{u}\right. \\
\left.+F\left(2 X+G_{u}\right) X_{x}+\left(F G_{u u}-F_{u} G_{u}-X F_{u}\right) U\right) W \\
+F U_{t}-F^{2} U_{x x}-F G_{u} U_{x}+2 F U X_{x}-U^{2} F_{u}=0
\end{array}
$$

By virtue of (13), this is identically satisfied given that a $W$ exists satisfying (19), which in turn gives that a $V$ exists satisfying (17), thus proving our claim.

\subsection{Nonlinear Wave Equation}

We now consider the nonclassical symmetries of (11b). Again, we set $T=1$. For this particular class of equations, it is necessary to consider two cases: (i) $X^{2} \neq F$ and (ii) $X^{2}=F$. Each will be considered separately.

Case (i) $X^{2} \neq F$

In this case, we have the following determining equations for the infinitesimals $X(t, x, u)$ and $U(t, x, u)$ : 


$$
\begin{gathered}
\left(X^{2}-F\right) X_{u u}+F_{u} X_{u}-2 X X_{u}^{2}=0 \\
\left(X^{2}-F\right)\left(2 X U X_{u u}+2 F X_{x u}+2 X X_{t u}-\left(F_{u}+2 X X_{u}\right) U_{u}-2 G_{u} X_{u}-F_{u u} U\right) \\
+\left(X^{2}-F\right)^{2} U_{u u}-2\left(2 X^{2} X_{t}+2 F X X_{x}-2 X U F_{u}+4 X^{2} U X_{u}\right) X_{u} \\
+2 X F_{u}\left(X_{t}+X X_{x}\right)-F_{u}^{2} U=0 \\
\left(X^{2}-F\right)\left(2 X U U_{u u}+2 X U_{t u}+2 F U_{x u}+U^{2} X_{u u}+2 U X_{t u}-F X_{x x}+X_{t t}\right) \\
-\left(X^{2}-F\right)\left(2 X_{u} U_{t}-2 F_{u} U_{x}+2 X_{t} U_{u}+G_{u} X_{x}-G_{u u}\right)-2 X\left(X_{t}^{2}-F X_{x}^{2}+U^{2} X_{u}^{2}\right) \\
-2\left(2 F X_{t}+2 F X X_{x}+2 X^{2} U X_{u}-X U F_{u}\right) U_{u}+U\left(F_{u} U-2 G_{u} X\right) X_{u} \\
-\left(4 X U X_{u}+2 G_{u} X-F_{u} U\right) X_{t}-\left(F_{u} X U+2 F G_{u}\right) X_{x}+F_{u} G_{u} U=0 \\
\left(X^{2}-F\right)\left(U^{2} U_{u u}+2 U U_{t u}+U_{t t}-F U_{x x}-F_{u} U_{x}\right) \\
-\left(U_{t}+U U_{x}\right)\left(2 X X_{t}+2 F X_{x}+2 X U X_{u}-F_{u} U\right) \\
+\left(2 F X_{t}+2 F X_{x}+2 F U X_{u}-F_{u} X U\right) U_{x}=0
\end{gathered}
$$

In the case of $G=0$, these determining equations appear in Näslund [38]. To calculate the nonclassical potential symmetries, we calculate the nonclassical symmetries for the associated system

$$
v_{t}=F(u) u_{x}+G(u), \quad v_{x}=u_{t}
$$

augmented with the two associated invariant surface conditions

$$
T u_{t}+X u_{x}=U, \quad T v_{t}+X v_{x}=V
$$

with $T=1$. This gives rise to two determining equations that have 43 and 44 terms, respectively. As we did in the previous section, we can simplify these determining equations. Solving (22) and (23) for $u_{t}, u_{x}, v_{t}$, and $v_{x}$ gives

$$
u_{t}=\frac{X G+U F-X V}{F-X^{2}}, \quad u_{x}=\frac{V-X U+G}{F-X^{2}}, \quad v_{t}=\frac{V-u X}{F-X^{2}}, \quad v_{x}=\frac{u}{F-X^{2}}
$$

Letting $V=\left(F-X^{2}\right) W+X U+G$, where $W=W(t, x, u, v)$ gives (24) as

$$
u_{t}=U-X W, \quad u_{x}=W, \quad v_{t}=F W+G, \quad v_{x}=U-X W
$$

Requiring compatibility through cross-differentiation gives rise to the following determining equations:

$$
\begin{gathered}
W_{t}+X W_{x}+U W_{u}+\left(X U+\left(F-X^{2}\right) W+G\right) W_{v}+\left(X_{u}-X X_{v}\right) W^{2} \\
+\left(X_{x}+U X_{v}-U_{u}+X U_{v}\right) W-U_{x}-U U_{v}=0 \\
X W_{t}+F W_{x}+\left(X U+\left(F-W^{2}\right) W\right) W_{u}+(F U+G X) W_{v}-U_{t}-U U_{u}-F U_{v} \\
+\left(F_{u}-X X_{u}+F X_{v}\right) W^{2}+\left(X_{t}+U X_{u}+G X_{v}+X U_{u}-F U_{v}+G_{u}\right) W=0
\end{gathered}
$$


To show that the nonclassical symmetries of (11b) are included in the nonclassical symmetries of (22) is to show that $W$ exists satisfying (26) if $X$ and $U$ satisfy (21). Eliminating derivatives of $W$ in (26) through cross-differentiation shows that (26) is compatible, provided that

$$
\left(X^{2}-F\right) A W^{3}-B W^{2}+C W-D=0,
$$

where $A, B, C$, and $D$ are precisely the expressions given in (21a)-(21d), thus showing that (27) is identically satisfied, again proving our claim.

Case (ii) $X^{2}=F$

For this special case, we will show the opposite is true. The nonclassical symmetries of the system are contained within the nonclassical symmetries of the single equation. For the system (22), we find determining equations give rise to $V=X U+G$, and that $U$ satisfies

$$
\begin{array}{r}
U_{t}-X U_{x}+U U_{u}+(G-X U) U_{v}=0 \\
U_{u}-X U_{v}+\frac{X}{2 X} U+\frac{G_{u}}{2 X}=0
\end{array}
$$

Compatibility of (28) by eliminating $U_{t}$ gives rise to the third equation

$$
X_{u} U_{x}-G_{u} U_{v}+\left(\frac{2 X X_{u u}-3 X_{u}^{2}}{4 X^{2}}\right) U^{2}+\left(\frac{X G_{u u}-2 X_{u} G_{u}}{2 X^{2}}\right) U-\frac{G_{u}^{2}}{4 X^{2}}=0
$$

Further compatibility between (28a) and (29) by eliminating all derivatives of $U$ gives rise to

$$
\left(2 X G_{u} X_{u u}-2 X X_{u} G_{u u}+G_{u} X_{u}^{2}\right) U^{2}+2 X_{u} G_{u}^{2} U+G_{u}^{3}=0
$$

If either $U_{t} \neq 0, U_{x} \neq 0$, or $U_{v} \neq 0$, then from (30) $G_{u}=0$ and (22) is linearizable via a hodograph transformation. Thus, the only case to consider is when $U=U(u)$. In this case, (28) can be solved, giving

$$
U=-c_{1}, \quad G=c_{1} X+c 2
$$

where $c_{1}$ and $c_{2}$ are arbitrary constants, and $X(u)$ is arbitrary.

We now turn our attention to the single Equation (11b). In the special case where $F=X^{2}$, we are restricted in the number of differential consequences of our invariant surface condition to be combined with our original PDE. Differential consequences of (12) (with $T=1$ and $X_{t}=X_{x}=0$ ) are

$$
\begin{aligned}
u_{t t}+X u_{t x}+X_{u} u_{t} u_{x} & =U_{t}+U_{u} u_{t} \\
u_{t x}+X u_{x x}+X_{u} u_{x}^{2} & =U_{x}+U_{u} u_{x}
\end{aligned}
$$

In the case where $X^{2} \neq F$, we can solve the original PDE (11b) along with differential consequence of the invariant surface condition (12) for $u_{t t}, u_{t x}$, and $u_{x x}$. In this special case where $X^{2}=F$, we can only solve for two second order derivatives of $u$. If we solve (11b) and (32b) for $u_{t t}$ and $u_{t x}$, the second determining equation in (32) becomes

$$
-U_{t}-U_{u} u_{t}+\left(X U_{u}+G_{u}\right) u_{x}+X_{u} u_{t} u_{x}+X X_{u} u_{x}^{2}=0
$$

and using the invariant surface condition (12), we obtain

$$
\left(2 X U_{u}+U X^{\prime}+G^{\prime}\right) u_{x}-U_{t}+X U_{x}-U U_{u}=0
$$


From (34) we see two cases emerge. If $2 X U_{u}+U X_{u}+G_{u}=0$, then $U_{t}-X U_{x}+U U_{u}=0$, and comparing with (28) shows they are identical if $U_{v}=0$. However, our analysis there showed the only solution is (31), and so the two results coincide. If $2 X U_{u}+U X_{u}+G_{u} \neq 0$, then we obtain the single determining equation

$$
\begin{array}{r}
\left(U_{t}-X U_{x}+U U_{u}\right)\left(2 X U_{t u}+2 X^{2} U_{x u}+2 X U U_{u u}+2 X X_{u u} U_{x}+2 X_{u} U U_{u}+X_{u u} U^{2}+G_{u u} U\right) \\
-\left(2 X U_{u}+X_{u} U+G_{u}\right)\left(U_{t t}-X^{2} U_{x x}+2 U U_{t u}+U^{2} U_{u u}-\left(2 X_{u} U+G_{u}\right) U_{x}\right)=0
\end{array}
$$

We make no effort to solve (35) in general; however, if $U=U(u)$, then (35) can be solved giving

$$
u=-\frac{G(u)+c_{1}}{X(u)+c_{2}}
$$

where $c_{1}$ and $c_{2}$ are arbitrary constants showing that the nonclassical symmetries of the single Equation (11b) contain the nonclassical symmetries of the equivalent system (22), and are in fact more general.

\section{3. $T=0$}

In applying the nonclassical method in the previous section, we assumed that $T \neq 0$, letting us set $T=1$ without loss of generality. We now consider the case when $T=0$. Without loss of generality, we can set $X=1$. Again, we will consider the nonlinear diffusion-convection and wave equations separately.

\subsection{Nonlinear Diffusion Equation}

In the case of the nonlinear heat Equation (11a), the nonclassical method gives rise to the following single equation for $U$ :

$$
U_{t}-F U_{x x}-2 F U U_{x u}-F U^{2} U_{u u}-\left(3 F_{u} U+G_{u}\right) U_{x}-2 F_{u} U^{2} U_{u}-F_{u u} U^{3}-G_{u u} U^{2}=0
$$

Applying the nonclassical method to the system (14) gives the single equation

$$
u_{t}-F\left(U_{x}+U U_{u}+u U_{v}\right)-F_{u} U^{2}-G_{u} U=0
$$

At this point, we set the coefficients of the derivatives to zero. This gives

$$
u_{t}=0, \quad F\left(U_{x}+U U_{u}+u U_{v}\right)+F_{u} U^{2}+G_{u} U=0
$$

showing that the only solutions to (14) are of the form $u=f(x)$. This was also noted in Bluman and Yan [24] in the case of $G=0$. However, we could continue to refine the nonclassical method and solve (38) for $u_{t}$ and impose compatibility with $u_{x}=U$. This would give

$$
\begin{array}{r}
U_{t}-F U_{x x}-2 F U U_{x u}-2 u F U_{x v}-F U^{2} U_{u u}-2 u F U U_{u v}-u^{2} F U_{v v} \\
-\left(3 F_{u} U+G_{u}\right) U_{x}-2 F_{u} U^{2} U_{u}+\left(G-u G_{u}-3 u F_{u} U\right) U_{v}-F_{u u} U^{3}-G_{u u} U^{2}=0
\end{array}
$$

Setting $U_{v}=0$ in (40) recovers (37), showing that a refinement in nonclassical method applied to the system (14) includes the nonclassical symmetries of the original Equation (11a). 


\subsection{Nonlinear Wave Equation}

In the case of the nonlinear wave Equation (11b), the determining equations are:

$$
\begin{aligned}
U_{u u} & =0 \\
U_{t u} & =0 \\
U_{t t}-F\left(U_{x x}+2 U U_{x u}+U^{2} U_{u u}\right)-2 F_{u} U^{2} U_{u} & \\
-\left(3 F_{u} U+G_{u}\right) U_{x}-F_{u u} U^{3}-G_{u u} U^{2} & =0
\end{aligned}
$$

For the system (22), they are

$$
\begin{aligned}
V_{t}+V V_{u}+(F U+G) V_{v}-F U_{x}-F U U_{u}-F V U_{v}-F_{u} U^{2}-G_{u} U & =0 \\
V_{x}+U V_{u}+V V_{v}-U_{t}-V U_{u}-(F U+G) U_{v} & =0
\end{aligned}
$$

Setting $U_{v}=0$ and requiring that (42) be compatible gives

$$
\begin{aligned}
U_{u u} V^{2}+2 U_{t u} V+U_{t t}-F & \left(U_{x x}+2 U U_{x u}+U^{2} U_{u u}\right)-2 F_{u} U^{2} U_{u} \\
& -\left(3 F_{u} U+G_{u}\right) U_{x}-F_{u u} U^{3}-G_{u u} U^{2}=0
\end{aligned}
$$

which by virtue of (41) shows that this is identically satisfied, proving that nonclassical symmetries of (11a) are included to those of (22).

\section{Conclusions}

In this paper, we have considered the symmetries of a nonlinear diffusion-convection and wave equation and equivalent systems. It is well known that classical Lie symmetries of differential equations and equivalent systems can be different. We question whether this is true if we extend the symmetries to include nonclassical symmetries. We have shown that in the majority of cases, the nonclassical symmetries of equivalent systems (sometimes termed potential symmetries) contain the nonclassical symmetries of the single equation counterpart. However, we have found a special case where the opposite is true, for the nonlinear wave equation when $X^{2}=F$, where we have found that the nonclassical symmetries of the single equation contain the nonclassical symmetries of a system equivalent. A natural question is whether this is true for more general equations

$$
\text { (i) } \begin{aligned}
u_{t} & =\left(F(t, x, u) u_{x}+G(t, x, u)\right)_{x} \\
\text { (ii) } u_{t t} & =\left(F(t, x, u) u_{x}+G(t, x, u)\right)_{x}
\end{aligned}
$$

There seems to be some indication that this is true, but further study is needed.

Acknowledgments: The authors B.A., S.B. and T.D. gratefully acknowledge the University of Central Arkansas for support through a graduate teaching assistantship.

Author Contributions: The authors contributed equally to this work.

Conflicts of Interest: The authors declare no conflict of interest.

\section{References}

1. Lie, S. Klassifikation und Integration von gewohnlichen Differentialgleichen zwischen $x, y$ die eine Gruppe von Transformationen gestatten. Math. Ann. 1888, 32, 213-281.

2. Arrigo, D.J. Symmetries Analysis of Differential Equations-An Introduction; Wiley: New York, NY, USA, 2015.

3. Bluman, G.; Kumei, S. Symmetries and Differential Equations; Springer: New York, NY, USA, 1989.

4. Olver, P.J. Applications of Lie Groups to Differential Equations, 2nd ed.; Springer: New York, NY, USA, 1993.

5. Ovsiannikov, L.V. Group properties of nonlinear heat equation. Dokl. AN SSSR 1959, 125, 492-495. 
6. Ovsiannikov, L.V. Group Analysis of Differential Equations; Academic Press: New York, NY, USA, 1982.

7. Bluman, G.W.; Reid, G.J.; Kumei, S. New classes of symmetries for partial differential equations. J. Math. Phys. 1988, 29, 806-881.

8. Bluman, G.W.; Cole, J.D. The general similarity solution of the heat equation. J. Math. Phys. 1969, 18, 1025-1042.

9. Bradshaw-Hajek, B.H.; Edwards, M.P.; Broadbridge, P.; Williams, G.H. Nonclassical symmetry solutions for reaction diffusion equations with explicit spatial dependence. Nonliner Anal. 2007, 67, 2541-2552.

10. Cherniha, R. New Q-conditional symmetries and exact solutions of some reaction- diffusion-convection equations arising in mathematical biology. J. Math. Anal. Appl. 2007, 326, 783-799.

11. Popovych, R.O.; Vaneeva, O.O.; Ivanova N.M. Potential nonclassical symmetries and solutions of fast diffusion equation. Phys. Lett. A 2007, 362, 166-173.

12. Bruzon, M.S.; Gandarias, M.L. Applying a new algorithm to derive nonclassical symmetries. Commun. Nonlinear Sci. Numer. Simul. 2008, 13, 517-523.

13. Arrigo, D.J.; Ekrut, D.A.; Fliss, J.R.; Le, L. Nonclassical symmetries of a class of Burgers' systems. J. Math. Anal. Appl. 2010, 371, 813-820.

14. Bluman, G.W.; Tian, S.F.; Yang, Z. Nonclassical analysis of the nonlinear Kompaneets equation. J. Eng. Math. 2014, 84, 87-97.

15. Cherniha, R.; Davydovych, V. Conditional symmetries and exact solutions of nonlinear reaction-diffusion systems with nonconstant diffusivities, Commun. Nonlinear Sci. Numer. Simulat. 2012, 17, 3177-3188.

16. Hashemi, M.S.; Nucci, M.C. Nonclassical symmetries for a class of reaction-diffusion equations: The method of heir-equations. J. Non. Math Phys. 2012, 20, 44-60.

17. Huang, D.J.; Zhou, S. Group-theoretical analysis of variable coefficient nonlinear telegraph equations. Acta Appl. Math. 2012, 117, 135-183.

18. Vaneeva, O.O.; Popovych, R.O.; Sophocleous, C. Extended group analysis of variable coefficient reaction diffusion equations with exponential nonlinearities. J. Math. Anal. Appl. 2012, 396, 225-242.

19. Broadbridge, P.; Bradshaw-Hajek, B.H.; Triadis, D. Exact non-classical symmetry solutions of Arrhenius reaction-diffusion. Proc. R. Soc. Lond. 2015, 471, doi:10.1098/rspa.2015.0580.

20. Louw, K.; Moitsheki, R.J. Group-invariant solutions for the generalised fisher type equation. Nat. Sci. 2015, 7, 613-624.

21. Pliukhin, O. Q-conditional symmetries and exact solutions of nonlinear reaction-diffusion systems. Symmetry 2015, 7, 1841-1855.

22. Yun, Y.; Temuer, C. Classical and nonclassical symmetry classifications of nonlinear wave equation with dissipation. Appl. Math. Mech. Eng. Ed. 2015, 36, 365-378.

23. Broadbridge, P.; Bradshaw-Hajek, B.H. Exact solutions for logistic reaction-diffusion equations in biology. ZAMP 2016, arXiv:1602.07370.

24. Bluman, G.W.; Yan, Y.S. Nonclassical potential solutions of partial differential equations. Eur. J. Appl. Math. 2005, 16, 239-261.

25. Gandarias, M.L.; Bruzon, M.S. Solutions through nonclassical potential symmetries for a generalized inhomogeneous nonlinear diffusion equation. Math. Meth. Appl. Sci. 2008, 31, 753-767.

26. Broadbridge, P.; White, I. Constant rate rainfall infiltration: A versatile nonlinear model 1. Analytic solution. Water Res. Res. 1988, 24, 145-154.

27. Rogers, C.; Stallybrass, M.P.; Clements, D.L. On two phase filtration under gravity and with boundary infiltration: Application of a Ba äcklund transformation. J. Nonliner Anal. Meth. Appl. 1983, 7, 785-799.

28. Katayev, I.G. Electromagnetic Shock Waves; Iliffe: London, UK, 1966.

29. Jeffery, A. Acceleration wave propagation in hyperelastic rods of variable cross-section. Wave Motion 1982, 4, 173-180.

30. Broadbridge, P.; Arrigo, D.J. All solutions of standard symmetric linear partial differential equations have classical Lie symmetry. J. Math. Anal. Appl. 1999, 234, 109-122.

31. Cherniha, R.; Serov, M. Symmetries, ansätze and exact solutions of nonlinear second-order evolution equations with convection terms. Eur. J. Appl. Math. 1998, 72, 21-39.

32. Arrigo, D.J.; Hill, J.M. Nonclassical symmetries for nonlinear diffusion and absorption. Stud. Appl. Math. $1995,72,21-39$. 
33. Arrigo, D.J.; Beckham, J.R. Nonclassical symmetries of evolutionary partial differential equations and compatibility. J. Math. Anal. Appl. 2004, 289, 55-65.

34. Niu, X.H.; Pan, Z.L. Nonclassical symmetries of a class of nonlinear partial differential equations with arbitrary order and compatibility. J. Math. Anal. Appl. 2005, 311, 479-488.

35. Wan, W.T.; Chen, Y. A note on nonclassical symmetries of a class of nonlinear partial differential equations and compatibility. Commun. Theor. Phys. 2009, 52, 398-402.

36. El-Sabbagh, M.F.; Ali, A.T. Nonclassical symmetries for nonlinear partial differential equations via compatibility. Commun. Theor. Phys. 2011, 56, 611-616.

37. Bluman, G.W.; Shtelen, V. Developments in similarity methods related to pioneering work of Julian Cole. In Mathematics Is for Solving Problems; Cook, S.L.P., Roytburd,V., Tulin, M., Eds.; SIAM: Philadelphia, PA, USA, 1996; pp. 105-118.

38. Näslund, R.N. On Conditional Q-Symmetries of Some Quasi-Linear Hyperbolic Wave Equations; Reprint Department of Mathematics, Lulea University of Technology: Lulea, Sweden, 2003.

(C) 2016 by the authors; licensee MDPI, Basel, Switzerland. This article is an open access article distributed under the terms and conditions of the Creative Commons Attribution (CC-BY) license (http://creativecommons.org/licenses/by/4.0/). 